\title{
New Diagnostic Methods and Treatment Recommendations in Primary Ciliary Dyskinesia
}

Author(s)

Affiliation(s)

Article

Information

\section{(1) Nagehan Emiralioğlu}

Hacettepe University Faculty of Medicine, Department of Pediatric Pulmonology, Ankara, Turkey

Article Type: Invited Review

Article Group: Pediatric Pulmonology
Received: 11.06 .2020

Accepted: 02.09.2020

Available Online: 30.09 .2020

Cite this article as: Emiralioğlu N. New Diagnostic Methods and Treatment Recommendations in Primary Ciliary Dyskinesia. J Pediatr Acad 2020; 1(2): 42-49.

\section{Abstract}

Primary ciliary dyskinesia (PCD) is a rare and genetically heterogeneous disease and clinically characterized by neonatal respiratory distress, organ laterality defects, persistent rhinosinusitis, chronic bronchitis, and eventually bronchiectasis. Currently, there is no single "gold standard" diagnostic test for PCD. PICADAR (Primary Ciliary Dyskinesia Rule) score is a guide to decide for further evaluation of diagnostic tests in PCD. European Respiratory Society (ERS) and American Thoracic Society (ATS) recommend diagnostic tests, including nasal nitric oxide (nNO), high-speed video analysis (HSVMA), transmission electron microscopy (TEM) and genetic testing. Cryo-electron tomography and immunofluorescence methods are new techniques recently performed by specialized centers and needs to be improved. Age at diagnosis for PCD changes according to awareness of disease and available diagnostic tests in different centers. Regular follow-up and multidisciplinary approach is important in the management of PCD. The main aim of the treatment is to prevent pulmonary exacerbations and slow the progression of the disease since there are no treatment approaches to correct the underlying cilia structure and its functions in PCD. Although, there are not enough randomized controlled trials for the treatment of PCD, recent treatments are usually based on to improve the mucociliary clearance. Early diagnosis with multidisciplinary management and nutritional advice could improve growth and delay disease progression leading to bronchiectasis and lung function impairment in PCD.

Keywords: Primary ciliary dyskinesia, ciliopathy, diagnostic tests, mucociliary clearance 


\section{Introduction}

Primary ciliary dyskinesia (PCD) is a rare disease with clinical and genetic heterogeneity and often inherited with autosomal recessive pattern, characterized by chronic lower and upper respiratory tract infections due to impaired ciliary motility. ${ }^{1,2}$ It was first described by Kartagener et al in 1936 as a triad of chronic sinusitis, bronchiectasis and situs inversus. After this definition, Afzelius suggested that patients with PCD has impaired cilia structure and mucociliary clearance due to immotile cilia. ${ }^{3}$ Recently, the definition of "immotile cilia syndrome" has been replaced by the definition of "primary ciliary dyskinesia", which is also associated with abnormal cilia movements besides immotile cilia. ${ }^{4,5}$

\section{Epidemiology}

The prevalence of primary ciliary dyskinesia is estimated to be between 1: 2000-1: 40000 according to last reports. ${ }^{1}$ Also the frequency of $P C D$ in Europe is estimated to be 1:10000-1: 20000. ${ }^{6}$ The most important reason for this situation is the absence of a standard diagnostic approach between different centers. In addition, it is known that the prevalence is high in populations where consanguineous marriages are high. ${ }^{7}$ PCD has mainly autosomal recessive inheritance pattern, however autosomal dominant and $\mathrm{X}$-linked transition have been rarely reported. ${ }^{2}$ The mutations identified in 45 genes so far is known to be the cause of the disease. ${ }^{1}$ Clinical studies and research in recent years have led to increased awareness of the disease; however, $30 \%$ of patients can not be diagnosed despite improvements in diagnostic methods and screening tests. ${ }^{4}$

\section{Normal cilia structure and function}

Respiratory cilias protect the airways against inhaled pathogens and allergens and cilias have an important role in the host defense. The density of cilias decrease from upper airways to lower airways, and no cilias are seen in the alveoli. The cilia microtubule structure is formed by the combination of $\alpha$ and $\beta$ tubulin monomers. The axonemal structure consists of nine peripheral microtubule pairs and one central microtubule pair $(9+2)$ or without the microtubule pair $(9+0)$ in the central. Cilia can be categorized into three groups as " $9+2$ " motile cilia (motor cilium) with the dynein arm, " $9+0$ " motile cilia (nodal cilium) with the dynein arm, and " $9+0$ " immotile cilia (sensory cilium) without the dynein arm. ${ }^{8}$ (Figure 1)

Motor cilias $(9+2)$ are located on the apical surface of the upper and lower airways, central nervous system, ependymal cells in the ventricles, sperm tail and fallopian tubes. The outer dynein arm (ODA) and the inner dynein arm (IDA) contribute to the formation of nine double microtubule structures around the central microtubule. The connection between the central pair and the peripheral microtubule is provided with "radial spoke" protein and the connection between peripheral microtubules is provided by "nexin" proteins. Mucociliary clearance enables to removal of mucus and bacteria.

Nodal cilia has a " $9+0$ " structure and functionally, this cilia has rotational motion and provides organ lateralization during embryogenesis. Mutations in the nodal cilia genes result in laterality defects including situs inversus and situs ambigus.

Sensory cilia has also " $9+0$ " structure with no dynein arm and they are localized in the epithelial cells of the kidneys, bile ducts, pancreas, chondrocytes, fibroblast smooth muscle and neurons. These cilias have roles in the cell signaling pathways. ${ }^{8}$

Mutations in the motile cilias (motor cilia and nodal cilia) are called motile ciliopathies and primary ciliary dyskinesia is the known motile ciliopathy in this group. Mutations in the sensory cilias cause diseases with multiple organ involvement called immotile (sensory) ciliopathies. They are classified as Retinitis pigmentosa, Bardet Biedl syndrome, Polycystic kidney disease, Nephonophtisis, Skeletal dysplasia (Jeune syndrome), Joubert syndrome and Cranioectodermal dysplasia. Recent reports suggested that immotile ciliopathies have also motile cilia dysfunction and similar clinical spectrum like PCD.9,10

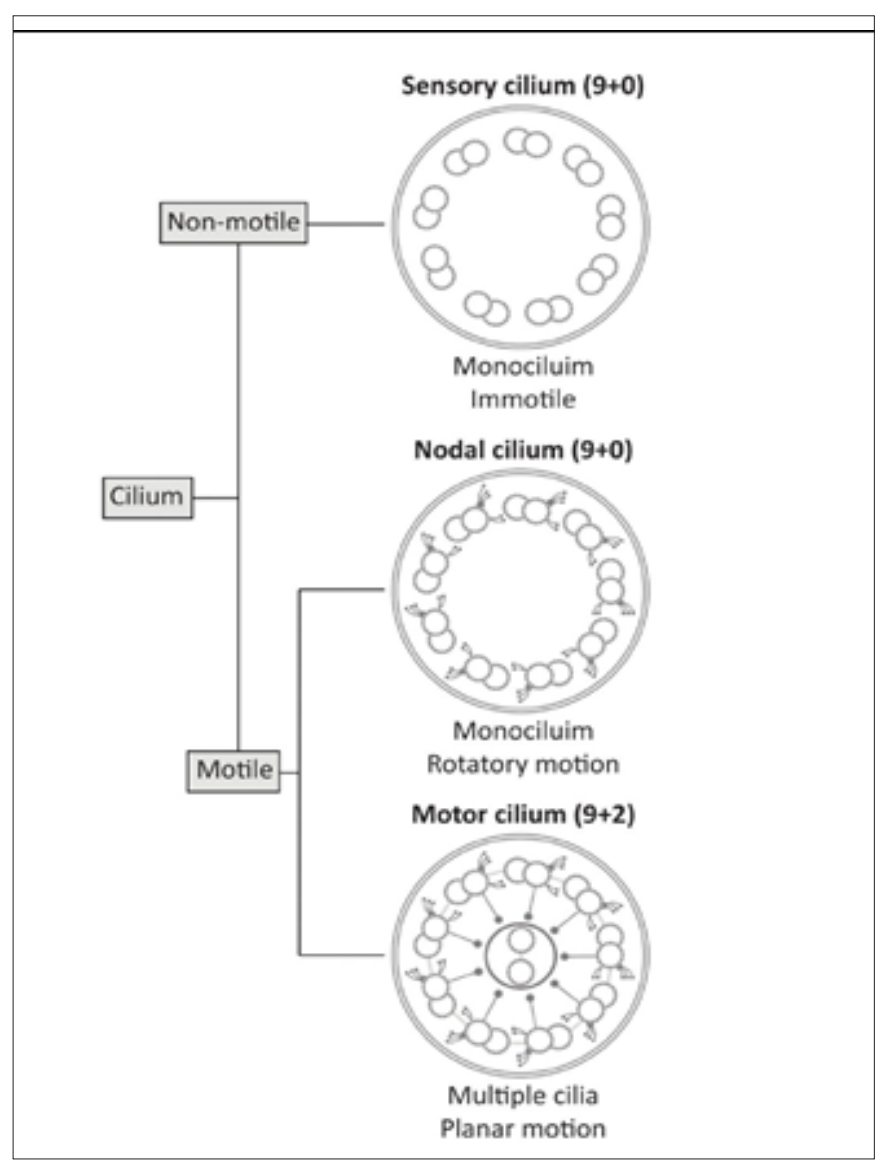

Figure 1. Motile and non-motile cilia types 


\section{Diagnosis}

The median age of diagnosis has been reported as 5.3 years in Europe and 2.6 years in UK, although it is younger in patients with situs inversus. 5,11 The diagnostic age changes according to awareness of disease and available diagnostic tests in different centers. Therefore, clinicians should suspect PCD in patients with typical clinical signs.

\section{Clinical findings}

Clinical findings are characteristic for patients with PCD. Abnormal structure and functions of motile cilia in the nasopharynx, middle ear, paranasal sinuses, lower airways and reproductive system cells are the cause of clinical findings in PCD. Symptoms can start at birth or develop in the first few months of life. Respiratory distress is observed over $80 \%$ of neonates despite term birth including history of mechanical ventilation or neonatal unit admission. ${ }^{12}$ Respiratory distress occurs mostly 12-24 hour after the birth, with no known cause. Chest X-ray shows atelectasis on different lobes. PCD should be suspected when the baby born on term and admits to hospital with unexplained respiratory distress, hypoxia and radiographic abnormalities such as atelectasis. ${ }^{9,13}$

Cilia ultrastructural defect and impaired cilia functions cause to decrease in the mucociliary clearance; therefore chronic productive cough is the most common reason to refer these patients. Persistant rhinorrhea or nasal congestion starting at first month of age is also a characteristic feature in almost $80 \%$ of the patients with PCD. ${ }^{9}$ Chronic rhinosinusitis, nasal polyp, recurrent acute otitis media, otitis media with effusion, chronic otitis media and conductive hearing loss are other common clinical findings in these patients. ${ }^{14,15}$

Recurrent bacterial infections in the lower airways eventually cause to bronchiectasis. Despite intensive medical treatments, PCD is generally a slow progressive disease, although some patients develop respiratory failure and lung transplantation is required. ${ }^{1}$

Situs inversus occurs in $40-50 \%$ and situs ambigus including heterotaxy occurs in $12 \%$ of patients with PCD. ${ }^{1}$ Complex congenital heart disease (6.2\%), esophageal atresia, biliary atresia are more common in patients with PCD compared with the normal population. ${ }^{16,17}$ Also hydrocephalus may be a sign in patients with PCD due to impaired cilia functions in the ependymal cells of ventricles. Respiratory symptoms are common in patients with heterotaxy (polyspleniaspleni) and complex heart defects. ${ }^{17}$ Other phenotypic features include pectus excavatum seen in $10 \%$ of cases and scoliosis seen in $5-10 \%{ }^{4}$

Since there are cilia in the sperm flagella nearly $100 \%$ of adult PCD male patients come with infertility. In women, the transition time of the ovum in the fallopian tubes is prolonged due to cilial dysfunction in the fimbria of fallopian tubes. Thus, males with PCD have diminished fertility due to reduced sperm motility, however females with PCD have increased risk of ectopic pregnancy because of abnormal fallopian tube transit of oocytes. ${ }^{9}$
In 2016, American Thoracis Society (ATS) defined four main most sensitive features to diagnose PCD. These are: Presence of laterality defect (OR 7.7); Unexplained respiratory distress lasting more than 24 hours in term newborn (OR 6.6); Early-onset (below 6 months) continuous nasal congestion (OR 3.4); Early-onset productive cough (OR 3.1). If there are at least three of these clinical characteristics, the specificity is over $96 \%$, and four of these clinical findings the specificity is over $98 \% .{ }^{18}$ In another study, Behan et al developed a scoring tool called "PICADAR (Primary Ciliary Dyskinesia Rule)" to predict the diagnosis of PCD. In this scoring, there are seven features, in addition to persistant productive cough including term born, neonatal chest symptoms, history of neonatal intensive care unit admission, chronic rhinitis, ear symptoms, situs inversus and congenital heart defect. The PICADAR score is a guide to decide for further evaluation of diagnostic tests in PCD. If the PICADAR score is 10 or more, probability of PCD is over $90 \%$, and if the score is 5 or more, patients should be evaluated for PCD. ${ }^{19}$ Table 1 shows which patients should refer for diagnostic tests of PCD according to ERS taskforce in 2017. ${ }^{15}$

\section{Table 1. \\ Which patients should undergo to diagnostic tests for Primary Ciliary Dyskinesia according to ERS Taskforce in $2017^{15}$}

Persistent wet cough, situs anomalies, congenital heart defect, persistent rhinitis, chronic otitis, hearing loss, unknown bronchiectasis, upper airway and lower airway symptoms in term newborn, patients who need intensive care unit in the neonatal period

Patients without a situs anomaly but having the above findings

Patients with a sibling with $\mathrm{PCD}$ and who have symptoms

Patients with PCD symptoms and high PICADAR scores

\section{Airway microbiology:}

Different microorganisms colonize the airways or infect the lungs. Therefore, respiratory tract cultures are obtained in 3-6 months intervals in patients with PCD in many centers. In childhood, Haemophilus influenza, Staphylococcus aureus, Moraxella catarrhalis and Streptococcus pneumonia colonization are common in the airways; Pseudomonas aeruginosa is common in young adults and is defined as the dominant microorganism in adult PCD patients. Non-tuberculosis mycobacteria are also seen in $15 \%$ of adults. ${ }^{12}$

\section{Pulmonary Function Tests}

In primary ciliary dyskinesia patients, as with non-CF bronchiectasis, progressive pulmonary obstruction develops with the progression of the disease. Unlike cystic fibrosis, the progression rate of the disease is slow in PCD. FEV1 shows an average decline of $0.8 \%$ per year. However, monitoring pulmonary functions is important in determining the treatment approach and prognosis. ${ }^{20}$ Goutaki et al reported that both growth and nutrition are affected adversely in PCD patients from early life and are both strongly associated with lung functions. ${ }^{21}$

\section{Radiological findings}

High resolution chest tomography (HRCT) is the most sensitive imaging method in the detection of bronchiectasis. However, it can not distinguish different 
causes of bronchiectasis, because the distribution of radiological findings change in different diseases. In primary ciliary dyskinesia, middle, lower lobe and lingula of the lungs are more involved and upper lobe involvement is seen later in the disease. Subsegmental atelectasis, peribronchial thickening, mucous plugging, air trapping, mosaic perfusion pattern, tree in bud pattern and ground glass appearance are shown in high resolution chest tomography, where structural changes in the pulmonary parenchyma begin in the infancy and childhood. ${ }^{22}$ (Figure 2)

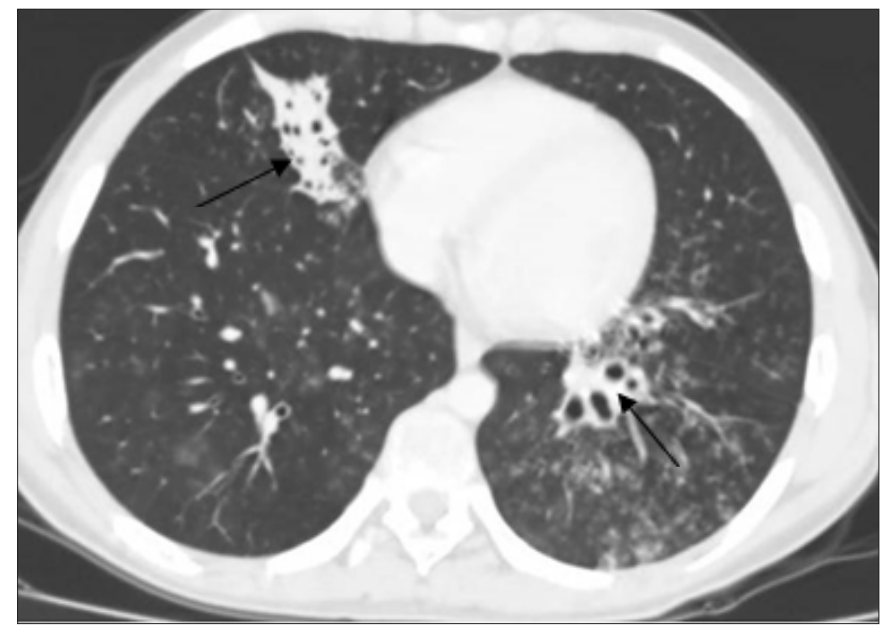

Figure 2a. Chest Computer Tomography Finding of Primary Ciliary Dyskinesia Patient- Bronchiectasis in the middle lobe of the right lung and ground glass appearance-consolidation - subsegmental atelectasis-bronchiectasis (arrow) in the lower lobe and lingula of the left lung

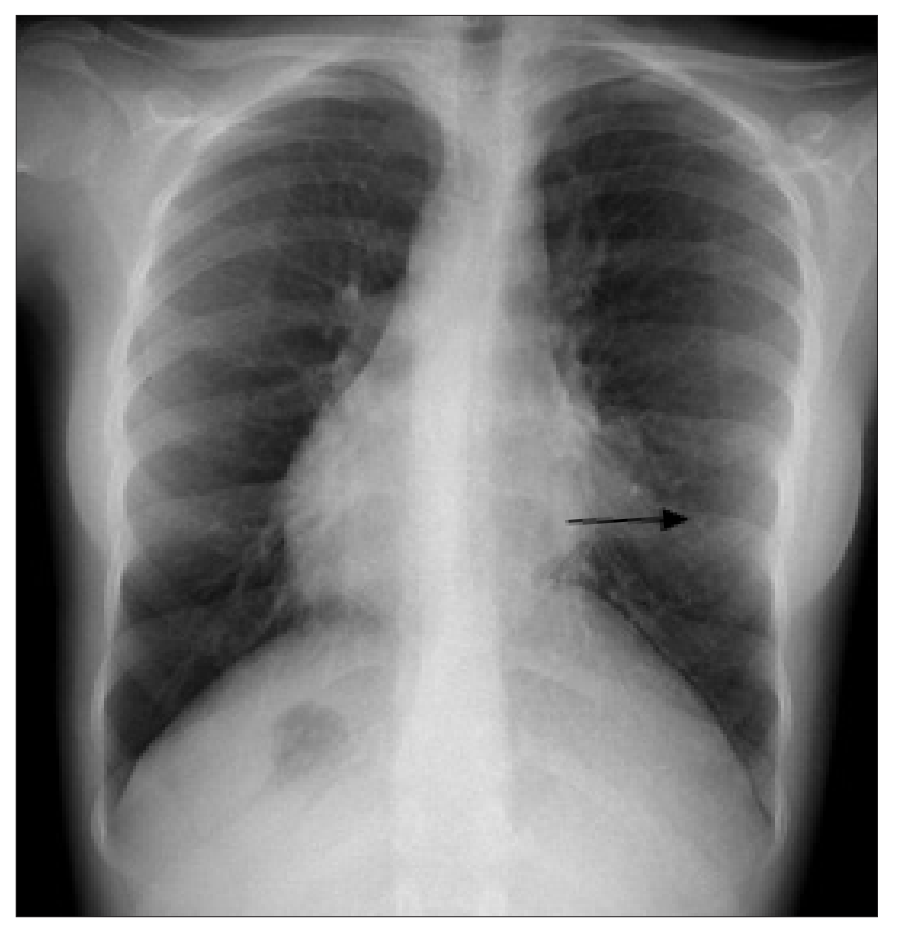

Figure 2b. Dextrocardia and atelectasis (arrow) on the chest radiograph of the patient with the diagnosis of Kartagener syndrome

\section{Diagnostic Tests in Primary Ciliary Dyskinesia}

European Respiratory Society (ERS) and American Thoracic Society (ATS) have published two evidence based guidelines in PCD diagnosis. However there is still no gold standard diagnostic test for PCD. Both of these guidelines recommend to use the combination of tests including nasal nitric oxide measurement, high speed video microscopy, immunofluorescence, transmission eletron microscopy and genotype analysis. ${ }^{15,23}$

\section{Screening Tests}

Nasal saccharin test, which was previously used as a screening test is not standardized and a subjective method, so it is not recommended for use as a screening test in PCD. Nasal saccharin tests and mucociliary clearance tests are not recommended since the false positivity rate is high in these methods. ${ }^{4,9}$

\section{Nasal nitric oxide measurement}

Nasal nitric oxide ( $\mathrm{nNO}$ ) levels are usually low in patients with $\mathrm{PCD}$, therefore $\mathrm{nNO}$ is used as a screening test according to ERS and ATS guidelines. ${ }^{15,23}$ Nasal NO measurement can be performed with tidal breathing or breath holding maneuver. However, lower values are usually obtained during the tidal breathing. NO measurement by chemiluminescence method and velum closure technique is recommended in patients over 6 years of age and nasal NO cutoff value of 77 $\mathrm{nL} / \mathrm{min}$ has $98 \%$ sensitivity, $99 \%$ specificity. ${ }^{15}, 24-26$ Measurement during tidal breathing is recommended in patients under 6 years of age with suspected PCD. It is used as a first-line test in patients with the diagnosis of PCD in most countries. ${ }^{15}$ Low $\mathrm{nNO}$ can also be detected in cystic fibrosis, nasal polyposis, upper respiratory tract infections and smokers. ${ }^{25}$ Nasal NO is usually normal in patients with mutations in few PCD-associated genes (RSPH1, GAS8, RPGR, CCNO, CDC103, CFAP221, DNAH9, FOXJ1, GAS2L2, LRRC56, NEK10, SPEF2, STK36, TTC12). Thus, $\mathrm{nNO}$ concentrations $>77 \mathrm{~nL} / \mathrm{min}$ do not exclude the diagnosis of PCD. ${ }^{27}$

\section{Diagnostic Tests}

Diagnostic tests are used to evaluate the ultrastructure and motility of motile cilias. Nasal samples obtained from the inferior nasal turbinate are preferred in the cilia evaluation, but samples can also be taken from the lower airways if bronchoscopy is performed. Epithelial cells can be obtained by curette, forceps or brushing methods. Nasal brushing method should be the first choice due to easy approach and samples should be obtained at least 2-4 weeks after the infection period. ${ }^{2}$

\section{High speed videomicroscopy analysis (HSVMA)}

In patients with suspected PCD, ERS recommends to use high speed videomicroscopy (HSVMA) including ciliary beat frequency and beat pattern examination for the diagnosis. ${ }^{15}$ The number of cilias, cilia beat frequency, beat pattern and efficiency of mucociliary clearance are evaluated with this method. ${ }^{1}$ Ciliary beat frequency was found 7-12 hz in the distal airways, 13$27 \mathrm{hz}$ in the trachea, main bronchus and nose. Biopsy samples are usually evaluated under a $37^{\circ} \mathrm{C}$ and inverted microscope. Specific beat patterns are classified as completely immotile, immotile with occasional residual movement, reduced bend and reduced beat amplitude, hyperfrequent with reduced amplitude or with circular motion. Mixed patterns may be observed in some situations. ${ }^{1}$ Exposure to air pollution, respiratory viral 
infections can cause non-specific ultrastructural changes and cilia dysfunction in the cilia. Secondary causes can be excluded by evaluating the samples of cilia at different times and culturing the cells in a sequential single layer / suspension system after biopsy. For definite diagnosis, high-speed videomicroscopy should be repeated after the cell culture. ${ }^{28,29}$ The sensitivity of HSVM in the diagnosis of PCD is $100 \%$, and its specificity is reported as $96 \%$. It is suggested that HSVMA is a reliable diagnostic test in experienced centers. ${ }^{30}$ However, in RSPH1, CCDC103, DNAH9, GAS8 mutations, HSVM may be normal. ${ }^{1}$

\section{Transmission electron microscopy}

Transmission electron microscopy should be used for the diagnosis of patients with suspected PCD according to ERS guidelines..$^{15}$ Electron microscopy provides the cilia ultrastructural evaluation to confirm the diagnosis. Although it was previously known as the gold standard method in the diagnosis of PCD, normal electron microscopy findings are observed in $30 \%$ of patients. ${ }^{31}$ In patients with a compatible history of PCD, further investigations should be carried out even electron microscopy is normal. However; there is no need for further diagnostic tests in patients with characteristic cilia ultrastructural defect suggesting PCD in electron microscopy. "Outer dynein arm defect, outer and inner dynein arm defect, microtubular disorganisation with inner dynein arm defect" are defined as hallmark diagnostic electron microscopy findings according to last ERS guideline. ${ }^{32}$ Electron microscopy findings including "central complex defect, mislocalization of basal bodies with few or no cilia, microtubular disorganisation defect with inner dynein arm, absent outer dynein arm from $25 \%-50 \%$ cross sections, combined inner and outer dynein arm absence from $25 \%-50 \%$ cross sections", indicate PCD diagnosis with other supporting evidence. However isolated inner dynein arm defect, compound cilia, naked cilia, membrane blebs, disorganized microtubuler structure are all secondary ciliary dyskinesia defects and usually disappear after the cell culture. ${ }^{32}$ Electron microscopy is frequently normal in "nexin link defects, central microtubule pair defects $(R S P H)$, cilia biogenesis defects (CCNO and MCIDAS), DNAH11, HYDIN mutations". ${ }^{1,2}$

\section{Cryo-electron tomography}

This method is a computed tomography adapted to electron microscopy. This method provides three dimensional evaluation of the cilia ultrastructureand demonstrates ultrastructural defects in patients with normal electron microscopy, such as DNAH11 and HYDIN mutations. ${ }^{33}$

\section{Immunofluorescence method}

Immunofluorescence method is based on the analysis of antibodies against axonemal proteins in the evaluation of abnormalities of the cilia ultrastructure. ERS recommends this method because it is cheaper and easier than the other tests. Immunofluorescence staining can detect PCD patients with normal ultrastructural evaluation. In addition to the outer dynein arm defects and microtubule disorganisation with the inner dynein arm defects that can be diagnosed by electron microscopy, it is also a useful method in abnormalities in the "nexin" connection and "radial spoke" defects. ${ }^{15}$ There are different antibody stainings (DNAH5, DNALI1, RSPH4A, RSPH9, RSPH1, GAS8) for many proteins developed for this purpose. ${ }^{1,2}$

\section{Genetic analysis}

ERS and ATS recommend genetic testing in patients with suspected PCD. ${ }^{15,23}$ It can be used to diagnose patients whocan notbe diagnosed by high-speed videomicroscopy, electron microscopy and immunofluorescence method. Genetic tests should be evaluated with clinical findings and other results. Bi-allelic pathogenic mutation or hemizygous X-linked mutation in a known gene will confirm the diagnosis. Allele segregation analysis to the family is important to confirm the genetic diagnosis. In a study from different clinical centers in the USA and Canada, $65 \%$ of 200 PCD patients have been reported to have a bi-allelic mutation. ${ }^{34}$ Almost $65-70 \%$ of PCD patients can be diagnosed with the next generation sequencing technology, so that the method will contribute to early diagnosis and treatment. 4,35 Genetic testing can not diagnose approximately $30 \%$ of patients and negative genetic testing does not exclude PCD. Genetic analysis is also important in giving the family genetic consultation. ${ }^{1}$ Genes related with ultrastructural defects in PCD are summarised in Table $2 .^{36}$

\section{Table 2. \\ Classification of subgroups according to genetic mutations in $P C D^{36}$}

\begin{tabular}{|c|c|}
\hline Cilia Ultrastructural Defect & Genes \\
\hline Normal ultrastructure & $\begin{array}{l}\text { DNAH11, CCDC164, CCDC65, } \\
\text { RPGR, OFD1 }\end{array}$ \\
\hline Outer dynein arm & $\begin{array}{l}\text { DNAH5, DNAl1, DNAI2, CCDC114, } \\
\text { CCDC151, ARMC4, DNAH1, TTC25, } \\
\text { TXNDC3, DNAL1, CCDC103 }\end{array}$ \\
\hline Outer and inner dynein arm & $\begin{array}{l}\text { DNAAF1, DNAAF2, DNAAF3, } \\
\text { HEATR2, LRRC6, SPAG1, } \\
\text { ZMYND10, DYX1C1, C21orf59, } \\
\text { CCDC103, PIH1D3 }\end{array}$ \\
\hline $\begin{array}{l}\text { Inner dynein arm and } \\
\text { axonemal organization }\end{array}$ & $C C D C 39, C C D C 40$, GAS8 \\
\hline $\begin{array}{l}\text { Central apparatus and } \\
\text { radial spoke }\end{array}$ & $\begin{array}{l}\text { RSPH1, RSPH3, RSPH4A, RSPH9, } \\
\text { HYDIN, DNAJB13 }\end{array}$ \\
\hline Absent or reduced cilia & CCNO, MCIDAS \\
\hline
\end{tabular}

Different genetic mutations affect the cilia ultrastructure, high speed videomicroscopic features and also clinical findings of patients in different ways. Situs abnormalities are seen in the ultrastructural defects affecting the dynein arm. Disorders in the cilia biogenesis (MCIDAS, CCNO), central pair (HYDIN), and radial spoke (RSPH1, $R S P H 4 A, R S P H 9$ ) do not cause situs abnormalities. Despite RSPH1 and DNAH9 mutations leading to mild clinical findings; CCNO, MCIDAS, CCDC39 and $C C D C 40$ mutations are associated with serious disease. Respiratory system findings appear early and are serious in mutations those with reduced cilia. Hydrocephalus is more common in CCNO and MCIDAS mutations. Nasal NO levels were found to be low in mutations causing loss of function in the motile cilia. ${ }^{1,37,38}$ 
In summary, diagnosis of PCD should be confirmed by at least two of the following methods in addition to the clinical features suggesting PCD: Abnormal high-speed videomicroscopy at least three times, abnormal electron microscopy findings, abnormal immunofluorescence examination findings, low nasal $\mathrm{NO}$, the biallelic mutations causing disease. ${ }^{2}$ According to ERS taskforce; in addition to clinical findings, suggestive electron microscopy findings for PCD or pathogenic biallelic mutations on genetic testing is necessary to define definitive PCD positive patients. However; in addition to clinical findings and low nasal NO, HSVM findings suggestive for $\mathrm{PCD}$ on three seperate occassions or HSVM findings suggestive for PCD following cell culture with normal electron microscopy is necessary to define highly likely PCD patients.

The limitations of the diagnostic tests are summarised in Table $3^{2,23}$

\section{Table 3.}

Limitations of Diagnostic Tests in PCD

\begin{tabular}{|c|c|}
\hline \multicolumn{2}{|l|}{ Diagnostic Test } \\
\hline Nasal NO & $\begin{array}{l}\text { It may also be low in other diseases such } \\
\text { as sinusitis, cystic fibrosis. } \\
\text { Sensitivity and specificity is higher in } \\
\text { patients older then } 5 \text { years old. } \\
\text { Nasal NO can be found normal in few } \\
\text { mutations (RSPH1, GAS8, RPGR, CCNO, } \\
\text { CDC103, CFAP221, DNAH9, FOXJ1, } \\
\text { GAS2L2, LRRC56, NEK10, SPEF2, } \\
\text { STK36, TTC12) }\end{array}$ \\
\hline $\begin{array}{l}\text { High speed } \\
\text { videomicroscopy }\end{array}$ & $\begin{array}{l}\text { Mild disorders can be considered as } \\
\text { normal. } \\
\text { Ciliary dyskinesia secondary to infection } \\
\text { and inflammation is common. In these } \\
\text { cases, discrimination between PCD and } \\
\text { secondary ciliary dyskinesia can be difficult. }\end{array}$ \\
\hline Electron microscopy & $\begin{array}{l}\text { Normal ultrastructural findings are detected } \\
\text { in } 30 \% \text { of cases. } \\
\text { It may cause a false positive diagnosis in } \\
\text { inner dynein arm defects. }\end{array}$ \\
\hline $\begin{array}{l}\text { Immunofluorescence } \\
\text { method }\end{array}$ & $\begin{array}{l}\text { It may be normal in } 20 \% \text { of cases. } \\
\text { When there is too much mucus in the } \\
\text { material, it becomes difficult to stain } \\
\text { technically. }\end{array}$ \\
\hline Genetic tests & $\begin{array}{l}\text { It is expensive due to the large number of } \\
\text { PCD genes ( } 45 \text { genes). } \\
\text { Genetic tests can identify about } 75 \% \text { of } \\
\text { cases. } \\
\text { New mutations in known genes should be } \\
\text { investigated in patients with suspected } \\
\text { PCD. }\end{array}$ \\
\hline
\end{tabular}

\section{Management of Primary Ciliary Dyskinesia}

\section{General management}

The main aim of the treatment is to prevent pulmonary exacerbations and slow the progression of the disease since there are no treatment approaches to correct the underlying cilia ultrastructural defect and ciliary dysfunction in PCD. ERS taskforce recommend to manage patients with definitive diagnosis of PCD and highly likely diagnosis of PCD to treat like PCD..$^{15}$ There are not enough randomized controlled trials for the treatment of PCD, treatments are usually based on the treatment of cystic fibrosis and non-cystic fibrosis bronchiectasis leading to improve mucociliary clearance. Patient education and airway cleaning techniques form the basis of the treatment. Prevention of smoking, protection from the air pollution, minimizing the exposure to respiratory pathogens, annual flu vaccines, pneumococcal polysaccharide vaccines and childhood routine vaccines are recommended. Body weight, height and body mass index (BMI) should be evaluated at each follow-up visit. Early diagnosis with multidisciplinary management and nutritional advice could improve growth and delay disease progression and lung function impairment in PCD..$^{1,39}$

\section{Pulmonary management}

Patients should be monitored in every 3-6 months. Chest $\mathrm{x}$-ray is recommended at the time of diagnosis; however it is not recommended in every follow-up visit. Since chest $x$-ray is not sensitive enough, it is not recommended other than the pulmonary exacerbation period. ${ }^{1,2}$ Chest CT is important in the early diagnosis of bronchiectasis. ${ }^{4}$ Chest CT and MRI have high compatibility in defining the bronchiectasis. Lung MRI can be used in the follow-up of PCD, middle and lower lobe involvement is common in these patients..$^{40}$ In addition, other radiological findings are peribronchial wall thickening, mucus plugging, bronchiectasis, atelectasis and bud tree appearance. ${ }^{2}$

Pulmonary function tests should be done in every 3-6 months. Lung clearance index $(\mathrm{LCl})$ can detect the lung pathology before the pulmonary function tests. Especially in microtubule defects, $\mathrm{LCl}$ is higher and FEV1 is lower. ${ }^{1,2}$

Sputum culture, cough swab or nasal swab should be taken 2-4 times in a year. For non-tuberculosis mycobacteria, a sputum sample should be taken every two years. Fungal culture should be obtained from cases unresponsive to treatment and patients should be evaluated in terms of Allergic Bronchopulmonary Aspergillosis. ${ }^{1,2}$

Different airway clearance techniques are recommended in PCD at least twice daily. Daily cardiovascular exercises, percussion techniques, manual chest physiotherapy techniques, positive pressure expiration methods increase clearance of secretions, regulate ventilation, increase exercise tolerance and reduce shortness of breath. ${ }^{1,2,4}$ Nebulized treatments may help patients to clear secretions, although evidence is not strong in PCD. In PCD, there was no difference on the pulmonary function tests, lower respiratory tract symptoms, sputum cytokines, inflammatory markers, quality of life scores at 3 months of treatment with $7 \%$ hypertonic saline compared to isotonic SF. ${ }^{41}$ Dornase $\alpha$ is used in CF that cleavages DNA released from neutrophils, reducing mucus viscosity and increasing airway clearance. However, in a study conducted in adults, inhaled dornase $\alpha$ treatment in non$\mathrm{CF}$ bronchiectasis has been shown to increase the rate of pulmonary exacerbation and cause a decrease in the respiratory functions..$^{42}$ There is no recommendation to use inhaled hypertonic saline, dornase $\alpha$ and also inhaled corticosteroids are not recommended if they have not asthma diagnosis in patients with PCD. 
Antibiotic treatments are recommended for symptomatic infections, prophylaxis, in $P$. aeruginosa eradication and chronic $P$. aeruginosa colonization. Pulmonary exacerbation is defined by the presence of three or more of the following seven items: 1) increased cough, 2 ) change in sputum volume and/or colour, 3) increased shortness of breath perceived by the patient or parent, 4) decision to start or change antibiotic treatment because of perceived pulmonary symptoms, 5) malaise, tiredness, fatigue or lethargy, 6) new or increased haemoptysis, and 7) temperature $>38^{\circ} \mathrm{C} .{ }^{43}$ Antibiotic treatments are decided based on the most common microorganisms $\mathrm{H}$. influenzea, S. pneumonia, M. catarrhalis, $S$. aureus in children and $P$. aeruginosa in older people. Treatment of asymptomatic infection is commonly changes according to clinican attitude. Although there is not strong evidence, it is recommended to treat the first isolates of pathogens with at least two weeks of antibiotic treatment. For $P$. aeruginosa, inhaled antibiotics are usually preferred and in some conditions oral antibiotics may be added. In chronic $P$. aeruginosa colonization inhaled antibiotics (colimycin, tobramycin, gentamycin) are usually recommended based on the CF literature. ${ }^{44,45}$

In patients with frequent pulmonary exacerbations, chronic or periodic oral or inhaled antibiotics reduce the pulmonary exacerbations and improve the quality of life of patients, also stabilizing their pulmonary functions. First multinational randomised controlled trial (BEST CILIA) in PCD showed that azithromycin maintenance therapy for 6 months was well tolerated and halved the rate of respiratory exacerbations. The authors concluded that azithromycin maintenance therapy is an option for patients with PCD with frequent exacerbations potentially leading to reduced need for additional antibiotic treatments and preventing irreversible lung damage. ${ }^{46}$

The role of thoracic surgery in patients with PCD is unclear and it is rarely indicated in PCD. Surgical resection therapy should be considered in localized lung disease, which causes serious symptoms, patients with frequent exacerbations, progressive with life-threatening hemoptysis despite medical treatment. ${ }^{47}$ Long-term results of patients with thoracic surgery are unknown. Thoracic surgery is not generally recommended in PCD as diffuse lung involvement decreases the success of localized surgical resection. In a multicentric study from different countries in Europe showed that patients who are lobectomized have lower FEV1, FVC z scores, and FEV1, FVC levels continue to decrease more than the non-lobectomy group after the surgery period. ${ }^{48}$ Lung transplantation is also an option in the end stage of lung disease. $^{1}$

\section{Upper airway management}

Because of many patients suffer from chronic rhinosinusitis, recurrent otitis media; ear, nose and throat examination 1-2 times a year and hearing test is recommended at least once a year. ${ }^{1,49}$

Nasal steroids, sinonasal rinsing with saline, nasal lavage with intermittent antibiotics and systemic antibiotics are used in the treatment. Surgical and nasal polypectomy can be applied to provide sinus drainage in patients resistant to medical treatment. Nasal surgery is not the first treatment option due to the risk of recurrence of nasal polyps..$^{2,49}$

In chronic upper respiratory diseases, conductive hearing loss and speech delay are observed in the long term period. Standard medical treatment is recommended in acute otitis media attacks. Adequate data are not available on surgical tympanostomy and ventilation tube management. ${ }^{1,2}$ ERS also recommends hearing aids in these patients rather than ventilation tubes. ${ }^{39}$

\section{Other system management}

Echocardiogram and abdominal ultrasound should be obtained in patients with PCD after the diagnosis. ${ }^{1}$

Compared to healthy controls in $\mathrm{PCD}$ patients; obstructive sleep apnea $(60 \%)$, attention deficit and hyperactivity are more common. ${ }^{1}$ This should be evaluated in the clinical history of PCD patients.

Infertility is common in men due to sperm immotility, assisted reproductive techniques with intracytoplasmic sperm injection are promising. In women, the transition time of the ovum to the fallopian tube is prolonged, but patients can have children with the invitro fertilization method. According to this; fertility tests should be obtained in adult PCD patients.

\section{Conclusion}

In primary ciliary dyskinesia, the awareness of the disease is low due to the symptoms and signs that are not specific to disease, and the difficulty in diagnostic tests cause delay in diagnosis. In the long-term evaluation, regular follow-up and multidisciplinary approach is important. Early diagnosis and treatment will slow chronic lung disease with bronchiectasis and positively affect the patients' quality of life.

Peer-review: Externally peer-reviewed.

Author Contributions: The author declare that she has participated in the design, execution, and analysis of the paper, and she has approved the final version.

Conflict of Interest: The author has no conflict of interest to declare.

Financial Disclosure: The author declared that this study has received no financial support.

\section{References}

1. Lucas JS, Davis SD, Omran H, Shoemark A. Primary ciliary dyskinesia in the genomics age. Lancet Respir Med. 2020;8:202216. [CrossRef]

2. Werner C, Onnebrink JG, Omran H. Diagnosis and management of primary ciliary dyskinesia. Cilia. 2015;4:2. [CrossRef]

3. Afzelius BA. A human syndrome caused by immotile cilia. Science. 1976;193:317-9. [CrossRef]

4. Lobo J, Zariwala MA, Noone PG. Primary ciliary dyskinesia. Semin Respir Crit Care Med. 201536:169-79. [CrossRef]

5. Knowles MR, Daniels LA, Davis SD, Zariwala MA, Leigh MW. Primary ciliary dyskinesia. Recent advances in diagnostics, genetics, and characterization of clinical disease. Am J Respir Crit Care Med. 2013;188:913-922. [CrossRef]

6. Kuehni CE, Frischer T, Strippoli MP, et al. Factors influencing age at diagnosis of primary ciliary dyskinesia in European children. 
Eur Respir J. 2010;36:1248-1258. [CrossRef]

7. Rumman N, Jackson C, Collins S, Goggin P, Coles J, Lucas JS. Diagnosis of primary ciliary dyskinesia: potential options for resource-limited countries. Eur Respir Rev. 2017;26:160058. [CrossRef]

8. Satir P, Christensen ST. Overview of structure and function of mammalian cilia. Annu Rev Physiol. 2007;69:377-400. [CrossRef]

9. Shapiro AJ, Zariwala MA, Ferkol T, et al. Diagnosis, monitoring, and treatment of primary ciliary dyskinesia: PCD foundation consensus recommendations based on state of the art review. Pediatr Pulmonol. 2016;51:115-132. [CrossRef]

10. Horani A, Ferkol TW. Primary ciliary dyskinesia and associated sensory ciliopathies. Expert Rev Respir Med. 2016;10:569-576. [CrossRef]

11. Rubbo B, Best S, Hirst RA, et al. Clinical features and management of children with primary ciliary dyskinesia in England. Arch Dis Child. 2020;105:724-729. [CrossRef]

12. Noone PG, Leigh MW, Sannuti A, et al. Primary ciliary dyskinesia: diagnostic and phenotypic features. Am J Respir Crit Care Med. 2004;169:459-467. [CrossRef]

13. Sagel SD, Davis SD, Campisi P, Dell SD. Update of respiratory tract disease in children with primary ciliary dyskinesia. Proc Am Thorac Soc. 2011;8:438-443. [CrossRef]

14. Sommer JU, Schäfer $K$, Omran $H$, et al. ENT manifestations in patients with primary ciliary dyskinesia: prevalence and significance of otorhinolaryngologic co-morbidities. Eur Arch Otorhinolaryngol. 2011;268:383-388. [CrossRef]

15. Lucas JS, Barbato A, Collins SA, et al. European Respiratory Society guidelines for the diagnosis of primary ciliary dyskinesia. Eur Respir J. 2017;49:1601090. [CrossRef]

16. Basu B, Brueckner M. Cilia multifunctional organelles at the center of vertebrate left-right asymmetry. Curr Top Dev Biol. 2008;85:151174. [CrossRef]

17. Kennedy MP, Omran H, Leigh MW, et al. Congenital heart disease and other heterotaxic defects in a large cohort of patients with primary ciliary dyskinesia. Circulation. 2007;115(22):2814-2821. [CrossRef] doi:10.1161/CIRCULATIONAHA.106.649038.

18. Leigh MW, Ferkol TW, Davis SD, et al. Clinical Features and Associated Likelihood of Primary Ciliary Dyskinesia in Children and Adolescents. Ann Am Thorac Soc. 2016;13:1305-1313. [CrossRef]

19. Behan L, Dimitrov BD, Kuehni CE, et al. PICADAR: a diagnostic predictive tool for primary ciliary dyskinesia. Eur Respir J. 2016;47:1103-1112. [CrossRef]

20. Santamaria F, Montella S, Tiddens HAWM, et al. Structural and functional lung disease in primary ciliary dyskinesia. Chest. 2008;134:351-357. [CrossRef]

21. Goutaki M, Halbeisen FS, Spycher BD, et al. Growth and nutritional status, and their association with lung function: a study from the international Primary Ciliary Dyskinesia Cohort. Eur Respir J. 2017;50:1701659. [CrossRef]

22. Kennedy MP, Noone PG, Leigh MW, et al. High-resolution CT of patients with primary ciliary dyskinesia. AJR Am J Roentgenol. 2007;188:1232-1238. [CrossRef]

23. O'Connor MG, Griffiths A, lyer NP, Shapiro AJ, Wilson KC, Thomson CC. Summary for Clinicians: Diagnosis of Primary Ciliary Dyskinesia. Ann Am Thorac Soc. 2019;16:171-174. [CrossRef]

24. American Thoracic Society; European Respiratory Society. ATS/ ERS recommendations for standardized procedures for the online and offline measurement of exhaled lower respiratory nitric oxide and nasal nitric oxide, 2005. Am J Respir Crit Care Med. 2005;171:912-930. [CrossRef]

25. Marthin JK, Nielsen KG. Choice of nasal nitric oxide technique as first-line test for primary ciliary dyskinesia. Eur Respir J. 2011;37:559-565. [CrossRef]

26. Leigh MW, Hazucha MJ, Chawla KK, et al. Standardizing nasal nitric oxide measurement as a test for primary ciliary dyskinesia. Ann Am Thorac Soc. 2013;10:574-581. [CrossRef]

27. Shapiro AJ, Davis SD, Leigh MW, Knowles MR, Lavergne V, Ferkol T. Limitations of Nasal Nitric Oxide Testing in Primary Ciliary Dyskinesia. Am J Respir Crit Care Med. 2020;202:476477. [CrossRef]

28. Raidt J, Wallmeier J, Hjeij R, et al. Ciliary beat pattern and frequency in genetic variants of primary ciliary dyskinesia. Eur Respir J. 2014;44:1579-1588. [CrossRef]

29. Hirst RA, Jackson CL, Coles JL, et al. Culture of primary ciliary dyskinesia epithelial cells at air-liquid interface can alter ciliary phenotype but remains a robust and informative diagnostic aid. PLoS One. 2014;9:e89675. [CrossRef]

30. Rubbo B, Shoemark A, Jackson CL, et al. Accuracy of High-Speed Video Analysis to Diagnose Primary Ciliary Dyskinesia. Chest. 2019;155:1008-1017. [CrossRef]

31. Boon M, Smits A, Cuppens $\mathrm{H}$, et al. Primary ciliary dyskinesia: critical evaluation of clinical symptoms and diagnosis in patients with normal and abnormal ultrastructure. Orphanet $J$ Rare Dis. 2014;9:11. [CrossRef]

32. Shoemark A, Boon M, Brochhausen $C$, et al. International consensus guideline for reporting transmission electron microscopy results in the diagnosis of primary ciliary dyskinesia (BEAT PCD TEM Criteria). Eur Respir J. 2020;55:1900725. [CrossRef]

33. Shoemark A, Hogg C. Electron tomography of respiratory cilia. Thorax. 2013;68:190-191. [CrossRef]

34. Genetic Disorders of Mucociliary Clearance Consortium(GDMCC). Available at: [CrossRef]

35. Horani A, Ferkol TW, Dutcher SK, Brody SL. Genetics and biology of primary ciliary dyskinesia. Paediatr Respir Rev. 2016;18:18-24. [CrossRef]

36. Horani A, Ferkol TW. Advances in the Genetics of Primary Ciliary Dyskinesia: Clinical Implications. Chest. 2018;154:645-652. [CrossRef]

37. Emiralioğlu N, Taşkıran EZ, Koşukcu C, et al. Genotype and phenotype evaluation of patients with primary ciliary dyskinesia: First results from Turkey. Pediatr Pulmonol. 2020;55:383-393. [CrossRef]

38. Davis SD, Rosenfeld M, Lee HS, et al. Primary Ciliary Dyskinesia: Longitudinal Study of Lung Disease by Ultrastructure Defect and Genotype. Am J Respir Crit Care Med. 2019;199:190-198. [CrossRef]

39. Barbato A, Frischer T, Kuehni CE, et al. Primary ciliary dyskinesia: a consensus statement on diagnostic and treatment approaches in children. Eur Respir J. 2009;34:1264-1276. [CrossRef]

40. Montella S, Santamaria F, Salvatore M, et al. Lung disease assessment in primary ciliary dyskinesia: a comparison between chest high-field magnetic resonance imaging and high-resolution computed tomography findings. Ital J Pediatr. 2009;35:24. [CrossRef]

41. Paff T, Daniels JM, Weersink EJ, Lutter R, Vonk Noordegraaf A, Haarman EG. A randomised controlled trial on the effect of inhaled hypertonic saline on quality of life in primary ciliary dyskinesia. Eur Respir J. 2017;49:1601770. [CrossRef]

42. O'Donnell AE, Barker AF, llowite JS, Fick RB. Treatment of idiopathic bronchiectasis with aerosolized recombinant human DNase I. rhDNase Study Group. Chest. 1998;113:1329-34. [CrossRef]

43. Lucas JS, Gahleitner F, Amorim A, et al. Pulmonary exacerbations in patients with primary ciliary dyskinesia: an expert consensus definition for use in clinical trials. ERJ Open Res. 2019;5:001472018. [CrossRef]

44. Wijers CD, Chmiel JF, Gaston BM. Bacterial infections in patients with primary ciliary dyskinesia: Comparison with cystic fibrosis. Chron Respir Dis. 2017;14:392-406. [CrossRef]

45. Crowley S, Holgersen MG, Nielsen KG. Variation in treatment strategies for the eradication of Pseudomonas aeruginosa in primary ciliary dyskinesia across European centers. Chron Respir Dis. 2019;16:1479972318787919. [CrossRef]

46. Kobbernagel HE, Buchvald FF, Haarman EG, et al. Efficacy and safety of azithromycin maintenance therapy in primary ciliary dyskinesia (BESTCILIA): a multicentre, double-blind, randomised, placebo-controlled phase 3 trial. Lancet Respir Med. 2020;8:493505. [CrossRef]

47. Smit HJ, Schreurs AJ, Van den Bosch JM, Westermann CJ. Is resection of bronchiectasis beneficial in patients with primary ciliary dyskinesia?. Chest. 1996;109(6):1541-1544. [CrossRef]

48. Kouis P, Goutaki M, Halbeisen FS, et al. Prevalence and course of disease after lung resection in primary ciliary dyskinesia: a cohort \& nested case-control study. Respir Res. 2019;20:212. [CrossRef]

49. Campbell R. Managing upper respiratory tract complications of primary ciliary dyskinesia in children. Curr Opin Allergy Clin Immunol. 2012;12:32-8. [CrossRef] 